\title{
Analyse de la réponse dynamique d'un système de suspension avec paramètres incertains
}

\author{
Dynamic Response Analysis of a Quarter Car Systems With Uncertainties
}

\section{Parameters}

\section{Fatma ABID ${ }^{1}$, Abdelkhalak ELHAMI ${ }^{2}$, Tarek MERZOUKI ${ }^{3}$, Lassaad WALHA ${ }^{4}$, Mohamed HADDAR $^{5}$}

\author{
${ }^{1}$ Laboratoire de Mécanique De Normandie, Institut National Des Sciences Appliquées De Rouen, Rouen, France, \\ fatma.abid@insa-rouen.fr \\ ${ }^{2}$ Laboratoire de Mécanique De Normandie, Institut National Des Sciences Appliquées De Rouen, Rouen, France, \\ Abdelkhalak.elhami@insa-rouen.fr \\ ${ }^{3}$ Laboratoire Ingénierie des Systèmes de Versailles, Université de Versailles Saint Quentin en Yvelines, Velizy, \\ France, tarek.merzouki@uvsq.fr \\ ${ }^{4}$ Laboratoire de Mécanique, Modélisation Et Productique, Ecole National D'ingénieurs De Sfax, Sfax, Tunisie, \\ walhalassaad@yahoo.fr \\ ${ }^{5}$ Laboratoire de Mécanique, Modélisation et Productique, Ecole national d'ingénieurs de Sfax, Sfax, Tunisie, \\ mohamed.haddar@enis.rnu.tn
}

\begin{abstract}
RÉSUMÉ. Dans cet article, une analyse d'incertitude paramétrique d'un système de suspension est proposée. Pour une évaluation raisonnable de son comportement dynamique, l'influence de l'incertitude de certains paramètres est prise en compte. Les équations dynamiques du système sont résolues en utilisant la méthode du chaos polynomial combinée avec le solveur ODE 45 de Matlab. L'effet de la perturbation aléatoire du ressort sur la réponse dynamique du système est discuté. Cette méthode est un outil efficace pour la propagation de l'incertitude. Pour obtenir plus de précision, les résultats du chaos polynomial sont comparés à des simulations directes.

ABSTRACT. In this paper, a parametric uncertainty analysis of a quarter car system is proposed. For a reasonable evaluation of its dynamic behavior, the influence of the uncertainty of some parameters is taken into account. The dynamic equations of the quarter car system are solved by using the polynomial chaos method combined with the ODE 45 solver of Matlab. The effect of the random perturbation of the spring on the dynamic response of the system is discussed. This method is an efficient tool for uncertainty propagation. To obtain more accuracy, the polynomial chaos results are compared with direct simulations.

MOTS-CLÉS. Système de suspension, Monte Carlo (MC), Polynôme de Chaos Généralisé (gPC).

KEYWORDS. Quarter car system, Monte Carlo (MC), Generalized Polynomial Chaos (gPC).
\end{abstract}

\section{Introduction}

The questionable quality of the road represents the main factor of discomfort, being directly responsible for accidents affecting not only the car components but also the security of passenger that causes serious injuries. In terms of road infrastructure, the low and the middle income countries are characterized by a higher accident rate [FLO 16]. Many scientific studies have investigated the dynamic behavior of quarter car system. All previous studies have presented the dynamic behavior of quarter car model considering deterministic parameters. However, the design tolerance and the manufacturer error incorporates the system into various uncertainty parameters. As a result, it becomes necessary to take into account uncertainty in order to ensure the robustness of the system [ SHA 15], [JIN 15].

In this context, several methods of uncertainties are developed in the literature, Monte Carlo (MC) simulation is well know technique in this field [FISH 96]. This method needs a great number of samples for reasonable accuracy. As a result, it is too costly. The polynomial chaos is presented as an efficient tool to deal with uncertainty propagation. This one is more attractive compared to other methods such as Monte Carlo [RUB 81]. Polynomial chaos represents the random input parameters 
variables as a probability distribution in a stochastic system governed by differential equations of motion.

The originality of this study is to investigate the effect of the uncertainty input of car quarter system. The objective is to investigate the capability of this method to determine the dynamic response of the mentioned system. This paper is organized as follows: After the introduction, we present the different stochastic approach such as Monte Carlo (MC) and generalized Chaos Polynomial (gPC). Next, the equation of motion for a two degrees of freedom are presented, After that, numerical results are presented. Finally, a conclusion was retrieved.

\section{Degree of freedom (DOF) of vehicle model}

The model used to apply the theory presented in the paper is shown in figure. 1 and is based on the two degrees of freedom where $m_{s}$ represents the sprung mass, $m_{u}$ represents the unsprung mass, $k_{s}$ and $k_{u}$ represent the non linear and linear spring of stiffness and $\mathrm{c}$ is a linear damper. An input was applied through a forcing function $\mathrm{z}(\mathrm{t})$. The differentional equations governing the system motion are given by:

$$
\begin{aligned}
& m_{s} \ddot{x_{1}}=-k_{s}\left(x_{1}-x_{2}\right)^{3}-c\left(\dot{x_{1}}-\dot{x_{2}}\right) \\
& m_{u} \ddot{x_{2}}=k_{s}\left(x_{1}-x_{2}\right)^{3}+c\left(\dot{x_{1}}-\dot{x_{2}}\right)+k_{u}\left(z(t)-x_{2}\right)
\end{aligned}
$$

The displacement $x_{1}(t)$ of the mass $m_{s}$ under uncertainty was analyzed using the gPC approaches and compared with results from a baseline standard Monte Carlo. Parametric uncertainty comes from the suspension stiffness. For such parameters, there is random variation within a range where the bounds are known from engineering estimates and a normal or uniform distribution can be used. The two springs considered to have uncertain spring constant values, uniformly or normally distributed about a mean stiffness value. This can be presented as follows:

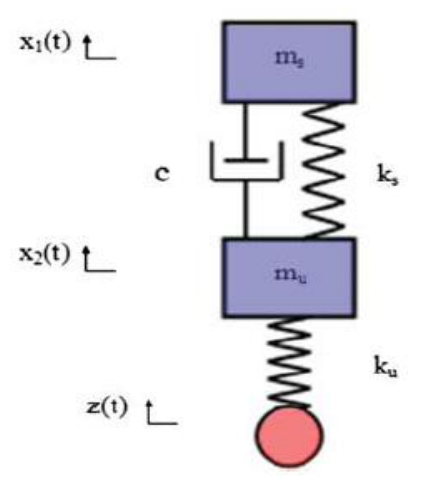

Figure 1. Quarter car model [KEW 12]

Displacement were expressed as a series expansion of Legendre polynomials of standard uniform random variables $\xi_{1}$ and $\xi_{2}$ or as a series expansion of Hermite polynomials were used when normal distribution was assumed. The system response was analyzed when subject to a sinusoidal terrain input with an amplitude of $0.1 \mathrm{~m}$ and a period $1 \mathrm{~s}$.

In general, the state may be represented as:

$X=\left[x_{1}, x_{2}, x_{1}, x_{2}\right]^{T}$ 
$x_{i}(t, \xi)=\sum_{j=0}^{P} x_{i, j}(t) \phi_{j}(\xi) \quad i=1,2$

$\dot{x}_{i}(t, \xi)=\sum_{j=0}^{P} \dot{x}_{i, j}(t) \phi_{j}(\xi) \quad i=1,2$

Where $\xi=\left[\xi_{1}, \xi_{2}\right]$.

The steps involved in the application of the Monte Carlo approach and gPC method to this system are discussed in the following section. Stiffness parameter values used here are given in table.1.

\begin{tabular}{|c|c|c|}
\hline Parameter & $\boldsymbol{\mu}$ & $\boldsymbol{\sigma}_{\boldsymbol{\mu}}\left(\mathbf{2} \boldsymbol{\sigma}_{\mathrm{N}}\right)$ \\
\hline $\mathbf{k}_{\mathrm{s}}$ & $40000 \mathrm{~N}$ & $4000 \mathrm{~N}$ \\
\hline $\mathbf{k}_{\mathrm{u}}$ & $15000 \mathrm{~N} / \mathrm{m}^{3}$ & $1500 \mathrm{~N} / \mathrm{m}^{3}$ \\
\hline $\mathbf{m}_{\mathrm{s}}$ & $750 \mathrm{~kg}$ & - \\
\hline $\mathbf{m}_{\mathrm{u}}$ & $50 \mathrm{~kg}$ & - \\
\hline $\mathbf{c}$ & $3000 \mathrm{Ns} / \mathrm{m}$ & - \\
\hline
\end{tabular}

Table 1. Parameters of quarter car model

\section{Stochastic approach}

\subsection{Monte Carlo method}

In this section, we will present the theory of Monte Carlo method. [SPA 08] and [FIS 96] give an exposition of this approach. This one provides successive resolutions of a deterministic system that incorporates random variables. This method is used when the problem to be dealt with is complex. It generates random samples for all uncertain parameters and according to their correlations and probability distributions. For each samples, a set of parameters is obtained and deterministic calculation following by numerical or analytic model is made. The main advantage of this technique is its ease of implementation. In fact, whatever the size and the complexity of a system, Monte Carlo technique can be implemented easily. The results of such a method are accurate. However, it often too costly since a reasonable accuracy require a large numbers of samples. In this study, the Monte Carlo is considered as a reference method in order to validate the efficiency of polynomial chaos method.

\subsection{Generalized Polynomial Chaos method (gPC)}

In this section, the generalized polynomial chaos (gPC) is described. An excellent exposition of this method is given by [SEP 15]. The chaos polynomial provides, for any stochastic process, a linear combination of orthogonal polynomials of random variables. Here, there is a review of mathematical approach. Any random variables $\left(X_{i}\right)$ can written as a second order random process. As a result, $\left(X_{i, j}\right)$ can be put in the following form [NEC 10],[NEC 11], [GUE 16] :

$$
X_{i, j}(x, \xi)=\sum_{j=0}^{\infty} \bar{X}_{i, j}^{-}(x) . \phi_{j}(\xi)
$$

Where $\xi$ is the vector of random variables. $\bar{X}_{i, j}$ is the deterministic component and $\phi_{j}(\xi)$ are the orthogonal polynomial functions such as Legendre, Hermite, etc... 
The orthogonal polynomials satisfy the orthogonality relation:

$$
\left\langle\phi_{1}, \phi_{\mathrm{j}}\right\rangle=\int \phi_{1} \phi_{\mathrm{j}} \mathrm{W}(\xi) \mathrm{d} \xi=\left\{\begin{array}{l}
0 \text { if } \mathrm{i} \neq \mathrm{j} \\
\left\langle\phi_{1}, \phi_{\mathrm{j}}\right\rangle \text { if } \mathrm{i}=\mathrm{j}
\end{array}\right.
$$

Where <.>is the internal product operator and $W(\xi)$ is the probabilistic density function (PDF). In practice, the gPC expansion is truncated to a finite order $N_{P}$ to limit the number of terms in the sum to a finite one. This truncation is indispensable to keep the problem feasible. The number of turn in the expansion depends on the order of polynomial chaos $\mathrm{p}$ and the dimensionality $\mathrm{n}$ of the random vector in the system :

$$
X_{i}(x, \xi)=\sum_{j=0}^{N_{p}} \overline{X_{i, j}}(x) \cdot \phi_{j}(\xi)
$$

With

$N_{P}=\frac{(p+r) !}{p ! r !}-1$

The calculation of representation by the gPC needs the determination of $N_{P}+1$ deterministic component called stochastic modes. Two approaches are used in order to determine such modes : an intrusive and non intrusive. The first approach consists in substituting random functions ,in the stochastic model, by their spectral representation, then, in making a projection called Galerkin [GHA 90],[GHA 93] of the resulting system based on gPC. The second approach consists in projecting directly the stochastic solution based on the gPC. Non intrusive approach prove to be more attractive for stochastic system in the sense that they need no modification or no manipulation of the system model. In the following, the non intrusive approach is presented. This one is attractive because it needs few calculations for the deterministic solutions in a finite number of points in the field of probabilistic variation. In order to determine these points, two methods are used: the spectral projection (NISP) and the regression method [BLA 08]. These ones are the main methods of the non intrusive gPC used in order to calculate stochastic modes.

In the NISP process [NEC 10], once the solution expressed in the base of polynomial chaos following the general expression, it is projected in this base which determines coefficients by:

$\bar{X}_{i, j}(x)=\frac{\left\langle X_{i}(x, \xi), \phi_{j}(\xi)\right\rangle}{\left\langle\phi_{j}(\xi), \phi_{j}(\xi)\right\rangle}=\frac{1}{\left\langle\phi_{j}{ }^{2}\right\rangle} \int X_{i}(x, \xi) \cdot \phi_{j}(\xi) \cdot W(\xi) d \xi, j=0, \ldots, N_{P}$

Different methods are used to calculate the integrals in equation such as Simpson methods, Gauss collocation techniques and numerical Monte Carlo methods. In the case of Gauss monodimensional integration, Eq. 10 can be expressed as following:

$\bar{X}_{i, j}(x)=\frac{1}{<\phi_{j}^{2}>} \sum_{k=1}^{Q} X_{i}\left(x, \xi^{k}\right) \cdot \phi_{j}\left(\xi^{k}\right) \cdot W\left(\xi^{k}\right), j=0, . ., N_{P}$

$W^{(k)}$ are the Gaussian integration weight while $\xi^{(k)}$ represent the Gaussian integration points also called Gauss Collocation points.

In the regression method, we calculate the stochastic modes in order to reduce the least squares sense, the difference $\varepsilon$ between the solution of the stochastic model and its approximation in the base of $\mathrm{gPC}$ is expressed as follows: 
$\varepsilon=\sum_{k=1}^{Q}\left[X_{i}\left(x, \xi^{(k)}\right)-\sum_{j=0}^{N_{P}} \overline{X_{i, j}}(x) \cdot \phi_{j}\left(\xi^{(k)}\right)\right]$

This technique needs a number, Q, of simulations higher than the number of stochastic coefficients:

$N_{P}+1<Q$

By designating $X_{i}=\left(X_{i}\left(x, \xi^{(1)}\right), \ldots, X_{i}\left(x, \xi^{(q)}\right)\right)$ the vector corresponding to the game simulations $\left\{\xi^{(q)}\right\} \quad, \bar{X}_{i, j}=\left(\bar{X}_{i, 0}, \ldots, \bar{X}_{i, N_{P}}\right)^{T}$ the vector of modal coefficients and Z the matrix of elements $z_{q, l}=\phi_{j}\left(\xi^{(q)}\right)$ such as the matrix $\left(Z^{T} Z\right)$ is non-singular. Then, the optimal solution of the classical least squares problem is expressed as follows:

$\bar{X}_{i, j}=\left(Z^{T} Z\right)^{-1} Z^{T} X_{i}$

\section{Simulations results}

In this section, the dynamic behavior of a quarter car model is investigated using the generalized Polynomial Chaos (gPC) method. The gPC results are compared with those obtained with the Monte Carlo (MC) method (1000 simulations). The gPC results are calculated using the ODE45 solver of software MATLAB. Both uniform and normal probability distributions are treated to describe the random parameter. The objective is to determine the most appropriate distribution of probability for this application.

\subsection{Uniform distribution of the uncertainty}

In this section, a uniform probability law is supposed to describe the dispersion of the non linear and linear spring of stiffness $k_{s}$ and $k_{u}$ :

$\phi_{1}(\xi)=\frac{k_{S_{\max }}+k_{S_{\min }}}{2}+\frac{k_{s_{\max }}-k_{S_{\min }}}{2} \xi$

$\phi_{2}(\xi)=\frac{k_{u_{\max }}+k_{u_{\min }}}{2}+\frac{k_{u_{\max }}-k_{u_{\min }}}{2} \xi$

Where $\xi$ is distributed uniformly within the orthogonally interval $\left[\begin{array}{ll}-1 & 1\end{array}\right]$.

The Legendre polynomials are the best used to deal with uniform uncertainties. The Legendre polynomials are written using the recurrence relation as follows:

$\left\{\begin{array}{l}(n+1) L_{n+1}(x)=(2 n+1) x L_{n}(x)-n L_{n-1}(x) \\ L_{0}(x)=1 \\ L_{1}(x)=x\end{array}\right.$

The standard deviation of the dynamic component of the sprung mass displacement $x_{1}(t)$ with uniformly distributed uncertain parameters is presented in Figure.2 for $\sigma_{k_{s}}=\sigma_{k_{u}}=20 \%$. The polynomial chaos results are compared with Monte Carlo simulations for 1000 operations. The standard deviation of the dynamic response of the sprung mass displacement which are predicted by gPC matches exactly with that of the Monte Carlo analysis. $\mathrm{p}=2$ is used in $\mathrm{gPC}$ model. This one is seen to be enough to capture the dynamic response of $x_{1}(t)$. 


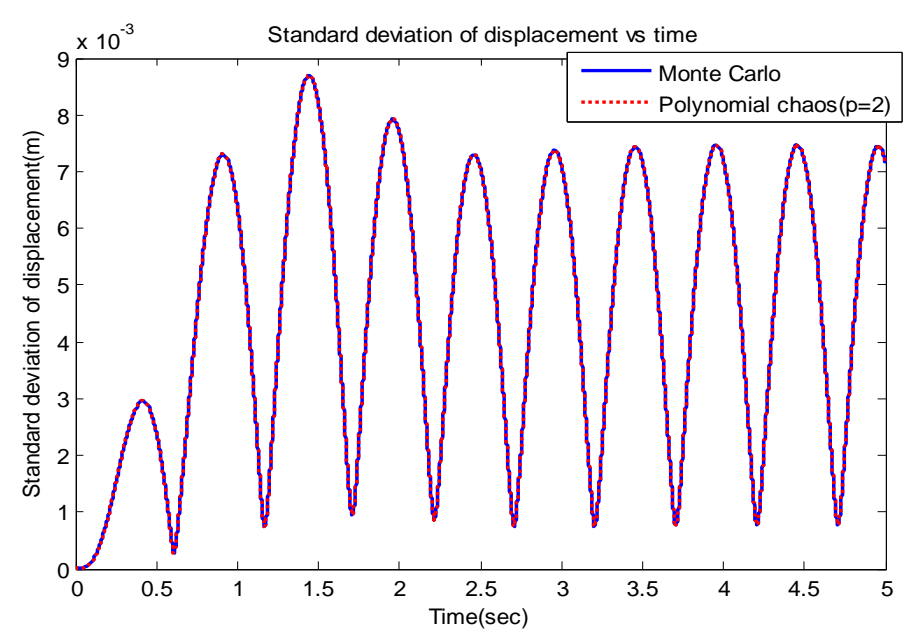

Figure 2. Standard deviation $20 \%$ of sprung mass displacement $x_{1}(t)$ when subject to step input (uniformly distributed uncertain parameters) $(p=2, n=1000)$

\begin{tabular}{|c|c|}
\hline Method & Ratio of simulation runs \\
\hline Monte Carlo(1000 runs) & $133.6601 \mathrm{~s}$ \\
\hline Polynomial chaos & $5,9975 \mathrm{~s}$ \\
\hline
\end{tabular}

Table 2. Computation time for the various approaches for system subject to step input (uniformly distributed uncertain parameters $)(p=2, n=1000)$

The results presented by table. 2 highlight the applicability of the gPC and show its efficient performance over Monte Carlo method in terms of reducing computational time.

\subsection{Normal distribution of the uncertainty}

In this part, a normal (Gaussian) probability law is supposed to describe the dispersion of the non linear and linear spring of stiffness $k_{s}$ and $k_{u}$ :

$k_{s}=k_{s 0}+\sigma_{k s} \xi$

$k_{u}=k_{u 0}+\sigma_{k u} \xi$

Where $\xi$ is a zero mean value Gaussian random variable, $k_{s 0}, k_{u 0}$ is the mean value of the non linear and linear spring of stiffness $k_{s}$ and $k_{u}$ and $\sigma_{k s}, \sigma_{k u}$ are the standard deviation of this parameter. In this case, the Hermite polynomials are the best used to deal with normal uncertainties. The Hermite polynomials are presented using the recurrence relation as follows:

$$
\left\{\begin{array}{l}
H_{0}(x)=1 \\
H_{n}(x)=x \cdot H_{n-1}(x)-\frac{d H_{n-1}(x)}{d x}
\end{array}\right.
$$

The standard deviation of the dynamic component of the sprung mass displacement $x_{1}(t)$ with normally distributed uncertain parameters is presented in Figure.2. The polynomial chaos results are compared with Monte Carlo simulations for 1000 operations. The standard deviation of dynamic response of the sprung mass displacement which are predicted by gPC matches exactly with that of the Monte Carlo analysis. The figure shows that the standard deviation with normal distribution of uncertain parameters has an amplitude less than the one of uniform distribution. 


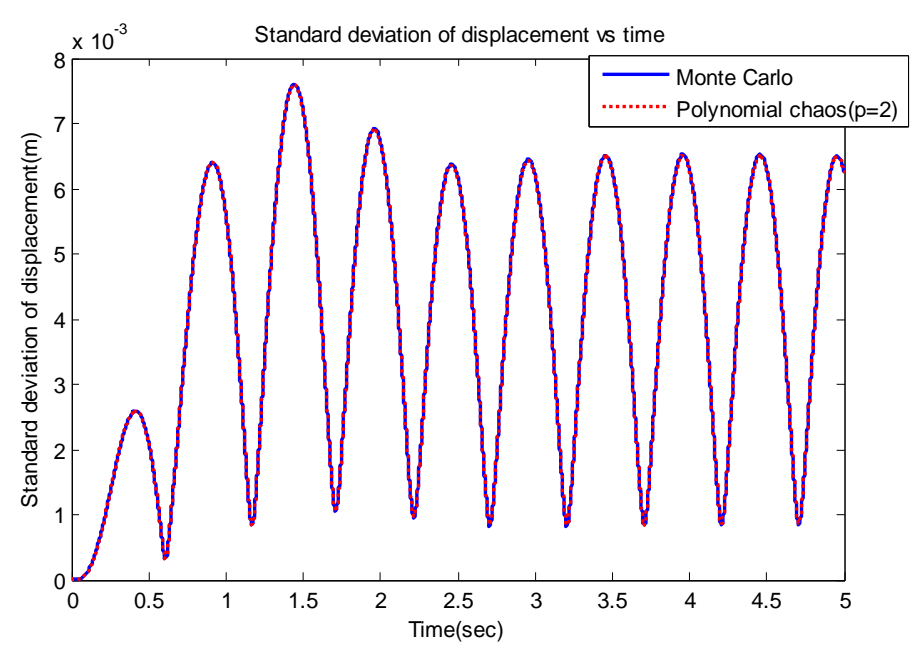

Figure 3. Standard deviation $20 \%$ of sprung mass displacement $x_{1}(t)$ when subject to step input (normally distributed uncertain parameters) $(p=2, n=1000)$

\begin{tabular}{|c|c|}
\hline Method & Ratio of simulation runs \\
\hline Monte Carlo(1000 runs) & $139.071 \mathrm{~s}$ \\
\hline Polynomial chaos & $6.0910 \mathrm{~s}$ \\
\hline
\end{tabular}

Table 3. Computation time for the various approaches for system subject to step input (normally distributed uncertain parameters $)(p=2, n=1000)$

The computational time for Monte Carlo and gPC corresponding to the result shown in Figure. 3 are given in table 3.The mean value and the standard deviation of the sprung mass displacement $x_{1}(t)$ have been calculated by the polynomial chaos method. The obtained results are compared with those given from Monte Carlo simulations for 1000 operations. The results are plotted in Figures. 4 and 5 for $\sigma_{k_{s}}=\sigma_{k_{u}}=20 \%$ and $\sigma_{k_{s}}=\sigma_{k_{u}}=40 \%$. These figures show that the obtained solutions oscillate around the Monte Carlo simulation which is the reference simulation. It can be observed that with a standard deviation $\sigma_{k_{s}}=\sigma_{k_{u}}=20 \%$ the polynomial chaos solutions provide a good accuracy comparing with Monte Carlo results. When the standard deviation increases, the error increases also.

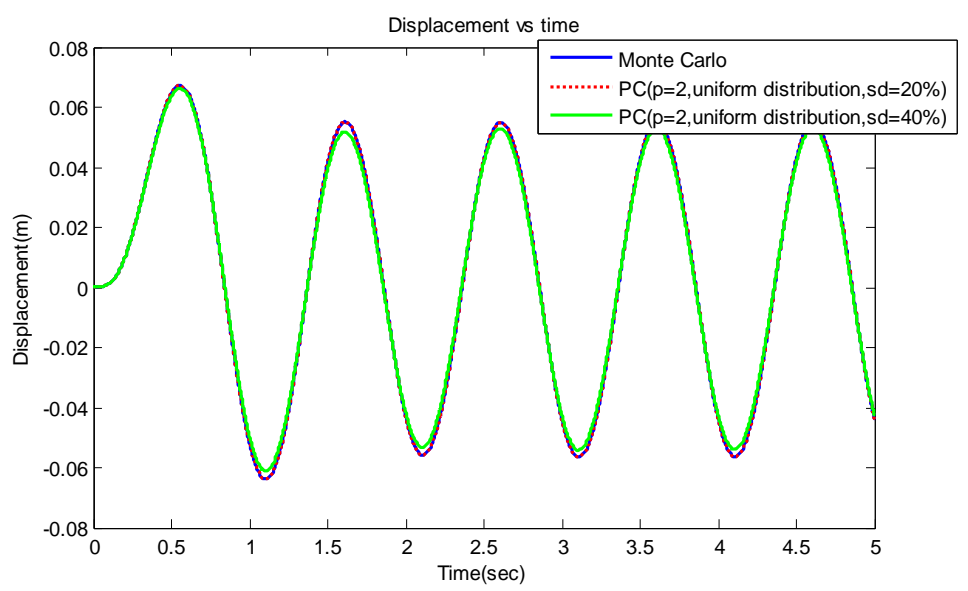

Figure 4. Mean value of sprung mass displacement $x_{1}(t)$ when subject to step input (uniformly distributed uncertain parameters) ( $p=2, n=1000, s d=20 \%, s d=40 \%)$ 


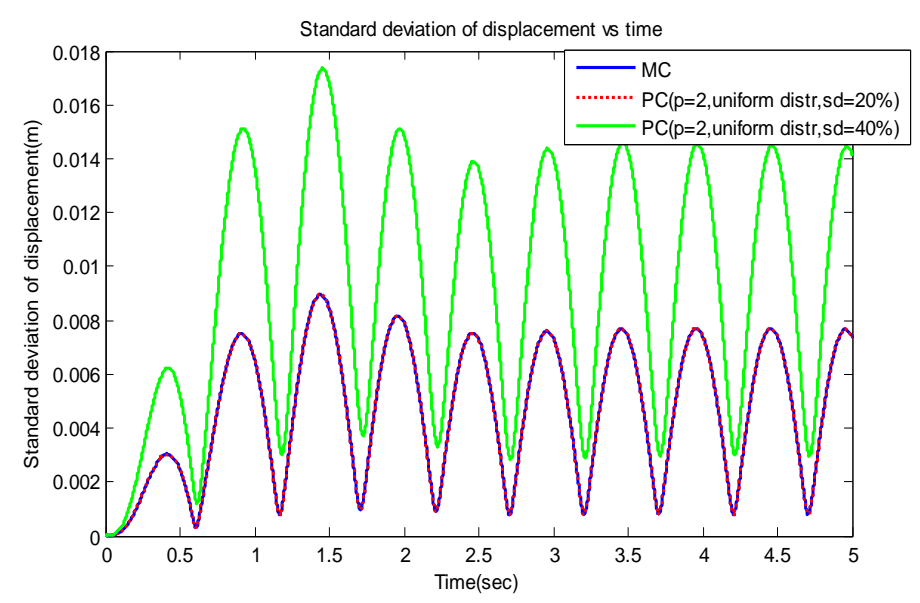

Figure 5. Standard deviation of sprung mass displacement $x_{1}(t)$ when subject to step input (uniformly distributed uncertain parameters) $(p=2, n=1000, s d=20 \%, s d=40 \%)$

\section{Conclusion}

In order to ensure the robustness of the dynamic response of a quarter car system, it is necessary to take into account uncertainties in the design. In this paper, we present the polynomial chaos method to study the mentioned system while considering the uncertainty in parameters of spring. The main results of the study shows that referring to Monte Carlo simulations, the polynomial chaos method is an efficient tool to model the dispersions of the quarter car parameters. An interest perspectives is to apply this method to a system with more degree of freedom.

\section{References}

[BLA 08] BLATMAN G., SUDRET B., «Sparse polynomial chaos expansions and adaptive stochastic finite. Compte Rendus Mécanique », vol. 336, no. 6, p. 518-523, 2008.

[FIS 96] FISHMAN G.S., «Monte Carlo, Concepts, Algorithms and Applications», First Ed. Springer Verlag, 1996.

[FLO 16] FLOREA A., COFARU L.L.,ROMAN L., COFARU N., «Applying The multiobjective optimization techniques in the design suspension systems», Journal of digital information management, vol. 14, p. 351-367, 2016.

[GHA 90] GHANEM R.G., SPANOS P.D., «Polynomial chaos in stochastic finite element», J. Appl. Mech., vol. 57, p. 197-202, 1990 .

[GHA 93] GHANEM R.G., SPANOS P.D., «A stochastic Galerkin expansion for nonlinear random vibration analysis», Probab. Eng. Mech., vol. 8, no. 3, p. 255-264, 1993.

[GUE 16] Guerine A., EL Hami A., Walha L., Haddar M., «A polynomial chaos method for the analysis of the dynamic behavior of uncertain gear friction system», Eur. J. Mech. A/Solids., vol. 59, p. 76-84, 2016.

[HUR 12] HURTADO J. E., ALVAREZ D. A., «The encounter of interval and probabilistic approaches to structural reliability at the design point», Comput. Methods Appl. Mech. Eng., vol. 225, p. 74-94, 2012.

[JIN 15] JINGYUE W., HAOTIAN W., LIXIN G., «Analysis of effect of random perturbation on dynamic response of gear transmission system», Chaos Solitons Fractals, vol.68 , p. 78-88, 2015.

[KEW 12] KEWLANI G., CRAWFORD J., IAGNEMMA K., «A polynomial chaos approach to the analysis of vehicle dynamics under uncertainty», Vehicle system dynamics, vol. 50, no 5, p. 749-774, 2012.

[NEC 10] NECHAK L., BERGER S., AUBRY E., «Robust analysis of uncertain dynamic systems: combination of the centre», WSEAS. Trans. Syst., vol. 9, no. 4, p. 386-395, 2010.

[NEC 11] NECHAK L., BERGER S., AUBRY E., «A Polynomial Chaos approach to the robust analysis of the dynamic behavior of friction systems», Eur. J. Mech. A, Solids, vol. 30, no. 4, p. 594-607, 2011.

[RUB 81] RUBINSTEIN R.Y., «Simulation and the Monte Carlo Method», John Wiley \& Sons Inc., New York, 1981. 
[SHA 15] SHA W., JINGSHAN Z., QINKAI H., FULEI C., «Dynamic response analysis on torsional vibrations of wind turbine geared transmission system with uncertainty», Renew. Energy, vol.78, p. 60-67, 2015.

[SEP 15] SEPAHVAND K., SCHEFFLER M., MARBURG S., «Uncertainty quantification in natural frequencies and radiated acoustic power of composite plates: analytical and experimental investigation», Appl. Acoust., vol. 87, p. $23-$ $29,2015$.

[SPA 08] SPANOS P. D., KONTSOS A., «A multiscale Monte Carlo finite element method for determining mechanical properties of polymer nanocomposites», Probab. Eng. Mech., vol. 23, no. 3, p. 456-470, 2008. 